\title{
High-gradient breakdown studies of an $X$-band Compact Linear Collider prototype structure
}

\author{
Xiaowei Wu, ${ }^{1,2}$ Jiaru Shi, ${ }^{1,2,4 *}$ Huaibi Chen, ${ }^{1,2}$ Jiahang Shao, ${ }^{1,2}$ Tetsuo Abe, ${ }^{3}$ \\ Toshiyasu Higo, ${ }^{3}$ Shuji Matsumoto, ${ }^{3}$ and Walter Wuensch ${ }^{4}$ \\ ${ }^{1}$ Department of Engineering Physics, Tsinghua University, Beijing CN-100084, China \\ ${ }^{2}$ Key Laboratory of Particle and Radiation Imaging, Tsinghua University, \\ Ministry of Education, Beijing CN-100084, China \\ ${ }^{3}$ KEK, High Energy Accelerator Research Organization, Tsukuba 305-0801, Japan \\ ${ }^{4}$ The European Organization for Nuclear Research, Geneva CH-1211, Switzerland
}

(Received 13 October 2016; published 8 May 2017)

\begin{abstract}
A Compact Linear Collider prototype traveling-wave accelerator structure fabricated at Tsinghua University was recently high-gradient tested at the High Energy Accelerator Research Organization (KEK). This $X$-band structure showed good high-gradient performance of up to $100 \mathrm{MV} / \mathrm{m}$ and obtained a breakdown rate of $1.27 \times 10^{-8}$ per pulse per meter at a pulse length of $250 \mathrm{~ns}$. This performance was similar to that of previous structures tested at KEK and the test facility at the European Organization for Nuclear Research (CERN), thereby validating the assembly and bonding of the fabricated structure. Phenomena related to vacuum breakdown were investigated and are discussed in the present study. Evaluation of the breakdown timing revealed a special type of breakdown occurring in the immediately succeeding pulse after a usual breakdown. These breakdowns tended to occur at the beginning of the rf pulse, whereas usual breakdowns were uniformly distributed in the rf pulse. The high-gradient test was conducted under the international collaboration research program among Tsinghua University, CERN, and KEK.
\end{abstract}

DOI: 10.1103/PhysRevAccelBeams.20.052001

\section{INTRODUCTION}

One of the highest priorities for the compact linear collider (CLIC) collaboration has been the development of high-gradient accelerating structures for the CLIC main linac [1]. The CLIC-G design, with waveguide damping, is the baseline for the CLIC main linac. Alternatives, such as choke-mode damped structure and detuned damped structure, are also being investigated [2-11]. Tsinghua University has been collaborating with CERN and KEK to assess the feasibility of $X$-band high-gradient accelerating structures not only for the CLIC project but also for the $X$-band XFELs and Thomson scattering sources. A structure based on previously tested CLIC prototype design was built and tested to implement high-gradient $X$-band technology at Tsinghua University to enable future developments. This structure allows the direct comparison of high-gradient performance and consequent validation of the fabrication technology. For this test, a structure called T24_THU_\#1 was chosen for the initial study on highgradient research [12]. The nomenclature "T24" refers to a

\footnotetext{
*shij@mails.tsinghua.edu.cn
}

Published by the American Physical Society under the terms of the Creative Commons Attribution 4.0 International license. Further distribution of this work must maintain attribution to the author(s) and the published article's title, journal citation, and DOI. standardized CLIC high-gradient test structure design. The T24 design has the same number of cells (i.e., 24), iris geometry, and iris tapering (i.e., " $T$ " in the name) as the CLIC baseline design, i.e., CLIC-G [2,3], but does not include the damping waveguides. The comparison of "T" and "TD," those with damping waveguides, allow high-field effects to occur in the iris and damping waveguide regions to be disentangled. Tests of an earlier series of test structures, i.e., T18 and TD18, have been reported previously $[2,13]$.

"THU" means that the structure was fabricated at Tsinghua University. The version discussed in this study operates at $11.424 \mathrm{GHz}$. The structure parameters of CLIC$\mathrm{G}$ and T24 are summarized in Table I, and further details about the rf design can be found in Refs. $[3,14,15]$. The modified Poynting vector, which is expressed as $S_{c}$, was reported in Ref. [16].

The high-gradient test reported here started on October 22, 2014 and ended on July 4, 2015 and was conducted in the New $X$-band Test Facility (Nextef) at KEK. Nextef [17] began operation in 2006, reusing equipment from Global Linear Collider Test Accelerator (GLCTA) [18]. Nextef provides up to $100 \mathrm{MW}$ for $X$-band accelerating structure studies.

CLIC high-gradient specifications are a loaded acceleration gradient of $100 \mathrm{MV} / \mathrm{m}$, a pulse width of $250 \mathrm{~ns}$, and a breakdown rate (BDR) of less than $10^{-7}$ per pulse per meter $[1,12,19]$. Previously tested prototype accelerating 
TABLE I. Structure parameters of CLIC-G, CLIC baseline design, and T24_THU_\#1, undamped test structure design.

\begin{tabular}{lcc}
\hline \hline Structure name & CLIC_G & T24_THU_\#1 \\
\hline Average unloaded gradient [MV/m] & 100 & 100 \\
Frequency [GHz] & 11.994 & 11.424 \\
Filling time [ns] & 67 & 57 \\
Total pulse length [ns] & 242 & 242 \\
First and last cell $Q$ factors $(\mathrm{Cu})$ & 5536,5738 & 6660,6990 \\
First and last cell shunt & 81,103 & 116,150 \\
$\quad$ impedances [M $\Omega / \mathrm{m}]$ & & \\
Number of regular cells & 26 & 24 \\
Peak input power [MW] & 41.7 & 42.4 \\
Maximum electric field [MV/m] & 196 & 226 \\
Maximum magnetic field [kA/m] & 393 & 255 \\
Maximum modified Poynting & 3.76 & 3.64 \\
$\quad$ vector [MW/mm ${ }^{2}$ ] & & \\
Maximum pulse heating & 33.1 & 13.8 \\
$\quad$ temperature rise [K] & & \\
\hline \hline
\end{tabular}

structures have achieved unloaded gradients in excess of $100 \mathrm{MV} / \mathrm{m}$ and as high as $120 \mathrm{MV} / \mathrm{m}$ in a test of an undamped structure [20,21].

The major limitation to the achievable gradient in roomtemperature accelerators is $\mathrm{rf}$ breakdown. Significant progress has been made in recent years in understanding the underlying physics of vacuum breakdown [16,22-33], but many open questions still remain. Well-controlled and documented high-gradient experiments are essential for increasing understanding of the phenomenon. During the high-gradient test described in this report, which extended over eight months, various measurements related to $\mathrm{rf}$ breakdown, such as the BDR, breakdown timing, and distribution of the longitudinal positions of the rf breakdowns along the structure length, were conducted. The measurements and new insights are presented in this report. This test was also aimed at verifying the assembling and bonding technology of the CLIC accelerator structures at Tsinghua University.

\section{FABRICATION, LOW-POWER RF TESTS, AND PREPARATION OF THE PROTOTYPE}

The disks and the coupler parts of the prototype were machined by the company VDL [34]. The parts were cleaned, etched, and bonded following procedures that are based on Global Linear Collider (GLC) fabricating technology [35,36]. The procedures included degreasing, cleaning by deionized water, and etching by hydrochloric acid liquor. In the preparation of the assembly of the prototype, the Tsinghua equipment and fabrication procedures were validated by bonding and mechanically measuring a test assembly that consisted of three cells. The CLIC baseline diffusion bonding procedure was used, which includes applying $0.1 \mathrm{MPa}$ pressure during bonding. The quality of the bonding joint

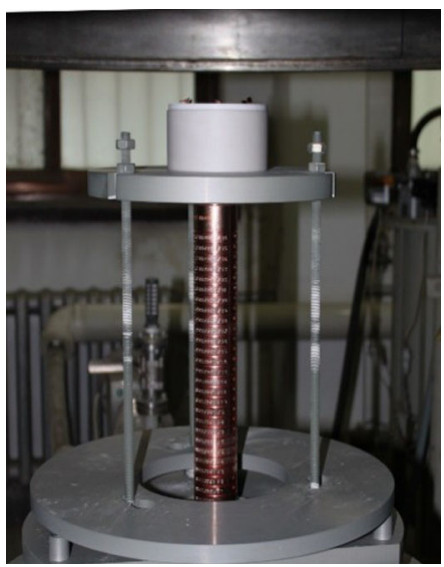

FIG. 1. Main cell stack for diffusion bonding in Tsinghua University.

was verified by cutting the assembly after bonding and confirming that grains crossed the bonding plane [37].

After successful completion of the bonding tests, the individual parts of the prototype were diffusion bonded in a hydrogen furnace at Tsinghua University, as shown in Fig. 1. Diffusion bonding of the main cell stack was performed at $1040^{\circ} \mathrm{C}$ [20]. The coupler parts and flanges were gold brazed to the main cell stack. Radio frequency measurements of the reflection coefficient were conducted before and after diffusion bonding. The resonant frequencies did not change. Then, tuning was performed in nitrogen flow at $25^{\circ} \mathrm{C}$. The structure was tuned to $11.424 \mathrm{GHz}$ at the working temperature of $30^{\circ} \mathrm{C}$, which is the standard cooling water temperature at Nextef. The tuning results are shown in Fig. 2.

Vacuum baking was performed in Tsinghua University. This step differed from the CLIC baseline procedure
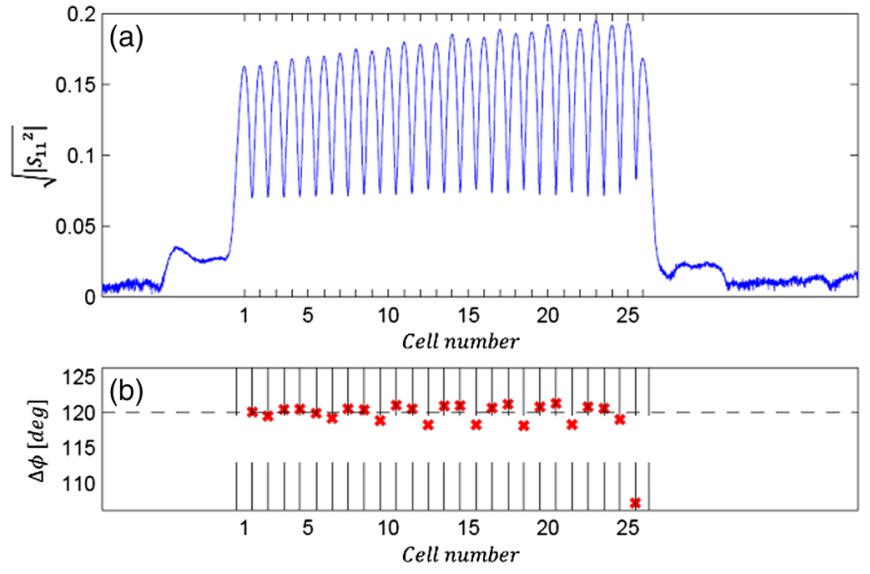

FIG. 2. Bead-pull measurement results after tuning the prototype at $11.424 \mathrm{GHz}$. (a) Electric field distribution along the beam axis ( $S_{11}$ is the reflection coefficient at the input port) and (b) phase advance per cell result. 


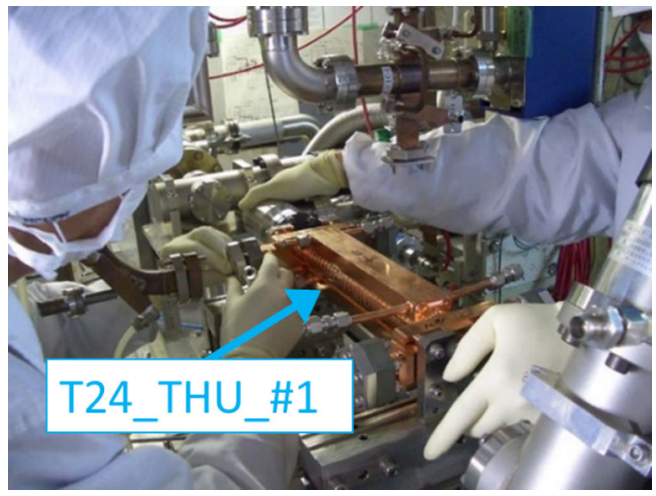

FIG. 3. Installation of the structure.

because of the temperature limitation in the vacuum furnace available at Tsinghua University. Vacuum baking was performed at $500^{\circ} \mathrm{C}$ for five days rather than the nominal baking parameters for CLIC structures, which is $650^{\circ} \mathrm{C}$ for seven days $[7,13]$. The structure was sealed by blind flanges with metallic gaskets, and the vacuum was pumped independently from the furnace during baking. The structure was kept under vacuum after baking by sealing with a valve and was shipped to KEK under vacuum.

Notably, the stainless-steel flanges turned light green after the gold brazing process. Water leakage into the furnace is speculated to cause this phenomenon. The effect will be investigated further.

The installation in Nextef was built by KEK staff following the standard KEK procedure [20], as shown in Fig. 3. Nitrogen gas flow from inside to outside the structure was maintained by overpressure during the opening of any part.

\section{HIGH-POWER TEST}

\section{A. Test stand}

The pulsed rf power of Nextef is produced by combining two $X$-band periodic permanent magnet focused klystrons and is capable of reaching $100 \mathrm{MW}$ with a repetition rate of $50 \mathrm{~Hz}$. The model of the klystron is E3768I provided by Toshiba. The pulse width is varied during the conditioning and testing processes. Measurements at the end of the test with 252 ns pulse width were conducted to allow direct comparison with the test results of other CLIC prototype structures. The high rf power is transferred into a concrete bunker where the test cavity is located. The forward, reflected, and transmitted powers are monitored by the directional couplers and used for breakdown detection in the operation system. The experimental setup is shown in Fig. 4. More details about the Nextef facility can be found in Refs. [17,20].

\section{B. Conditioning strategy}

Conditioning is the process of gradually improving the structure surface and gradient holding capability through the application of rf [22]. The conditioning operation was started with the rf power of the order of hundreds of kilowatts and the shortest pulse width of $51 \mathrm{~ns}$, which were progressively increased to the nominal peak power and the pulse width. The pulse width was kept constant for extended periods, and the power was ramped by a step, which ranged from 0.1 to $0.4 \mathrm{MW}$, until it exceeded the nominal maximum unloaded accelerating gradient of $E_{\text {acc }}=100 \mathrm{MV} / \mathrm{m}$. The pulse width was lengthened in approximately $50 \mathrm{~ns}$ steps. Then, input $\mathrm{rf}$ power was ramped from a few hundred kilowatts again for the new pulse width. The entire operation was divided into several

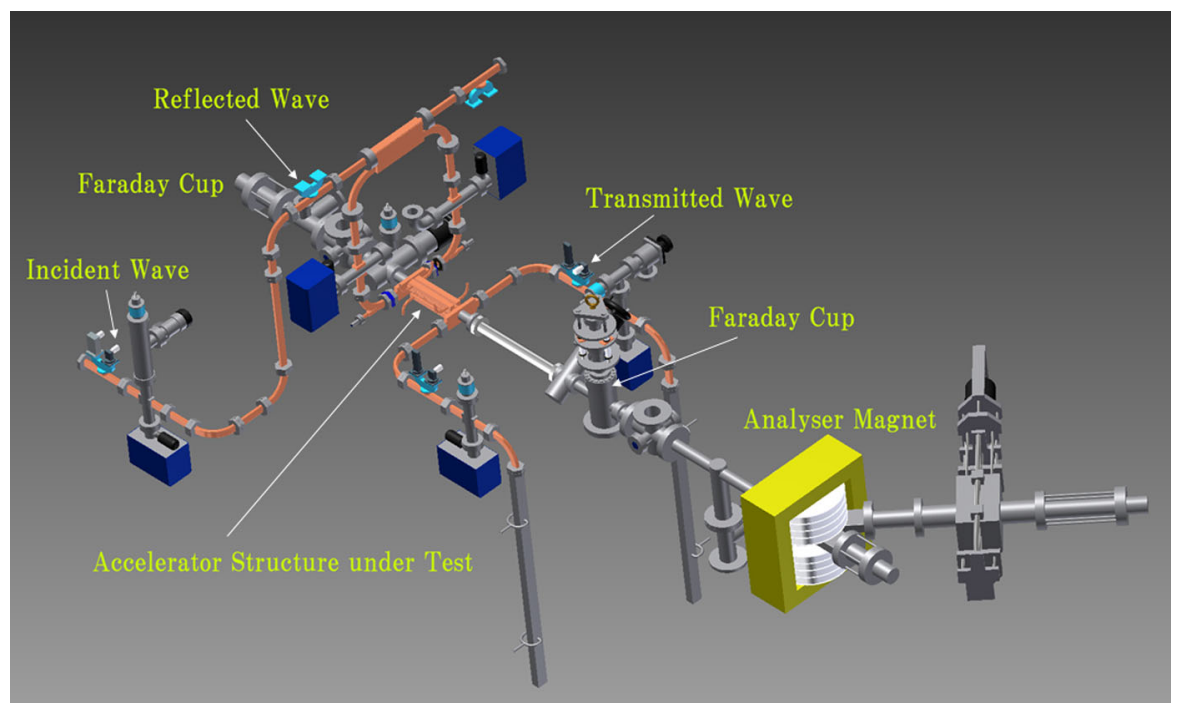

FIG. 4. Nextef accelerator testing setup. The incident, reflected, and transmitted waves are all measured by directional couplers. The waveguide distance from the structure input coupler to the directional coupler for the incident wave is approximately $2.2 \mathrm{~m}$. The distances from the structure beam pipe flange to the Faraday cups (i.e., Faraday cup UP and Faraday cup DN) are both 0.8 m. 
tens of "runs" to keep the data file size below a reasonable level for easy handling. Each conditioning and measurement run usually lasted for a few hundred hours.

Diagnostics used in the experiment include three directional couplers to monitor the input, reflected, and transmitted rf signals and two Faraday cups to collect upstream and downstream dark currents during rf conditioning. These signals were monitored pulse by pulse for breakdown detection $[17,38,39]$. Normal rf pulse signal and typical breakdown signal are shown in Fig. 5. The interlock system inhibited the subsequent pulse when any jump in reflected rf signal or Faraday cup was detected. Then, the system paused for several tens of seconds before the subsequent rf pulse, which is sufficiently long to allow the gas pressure to recover and to save the waveforms. These interlock events might indicate a breakdown event and were checked during offline data analysis [20]. The system reduced the rf power by approximately 5\% after breakdown detection and ramped the power up again by increasing $0.2 \mathrm{MW}$ over a period of $20 \mathrm{~s}$.

From the analysis of the breakdown time sequence, two types of breakdown events, i.e., normal breakdown (NL-BD), which occurred after operation over many pulses without breakdown, and "following-pulse breakdown" (FP-BD), which occurred at the first pulse following the breakdown pulse, were observed. The FP-BDs were triggered at the lower gradient than the previous breakdown because operation was restarted at a 5\% lower power level than the previous operational value. Similar sequences of follow-up breakdown events, such as "spitfest" and "chain breakdowns," have been reported in Refs. [24,40,41]. Given that FP-BDs occurred at a lower power level than

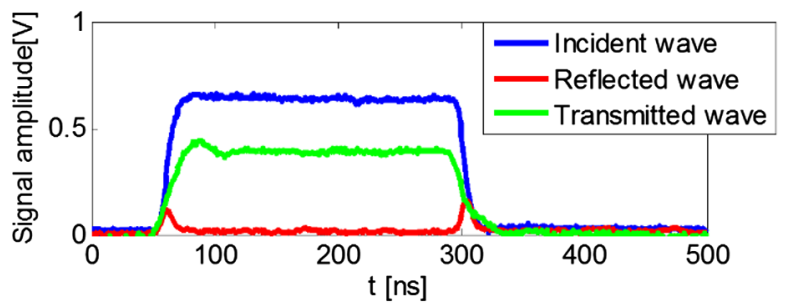

(a)

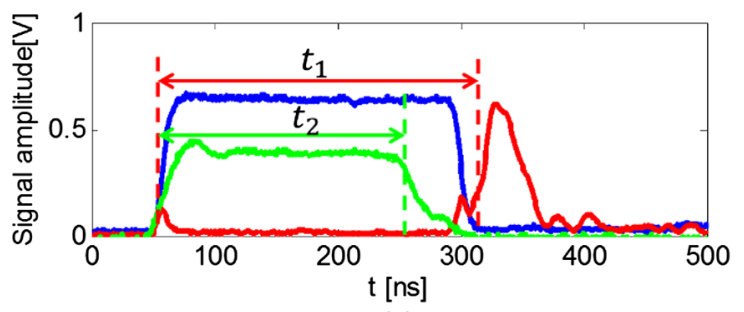

(b)

FIG. 5. Detected signals in Nextef. (a) Normal rf pulse signal and (b) typical breakdown signal. $t_{1}$ is the rising edge of the reflected wave, whereas $t_{2}$ is the falling edge of the transmitted wave. $t_{1}$ and $t_{2}$ are used for breakdown cell location and breakdown timing calculation. the initial breakdown, they are not used in this study for the evaluation of the breakdown number and BDR, unless explicitly stated otherwise. The unique characteristics of the FP-BDs will be discussed subsequently in this work.

\section{TEST RESULTS}

\section{A. Conditioning history of the prototype}

The summary of the conditioning history is shown in Fig. 6. The blue, green, and red points represent $E_{\text {acc }}$, the pulse width of rf power, and the accumulated number of breakdowns, respectively, as a function of the number of pulses. The $E_{\text {acc }}$ value was recorded at every interlock event, including BD and non-BD interlocks (which can be generated by the stopping operation manually). The dots that fall below the envelope of $E_{\text {acc }}$ correspond to interlocks occurring during the power ramping stage after the previous breakdown. The total rf-on time was approximately $3600 \mathrm{~h}$, during which time $6.47 \times 10^{8} \mathrm{rf}$ pulses occurred.

\section{B. BDR evaluation}

Dedicated runs, which were employed to evaluate the evolution of the BDR, were conducted 15 times during the course of the experiment. These runs provide key information of the conditioning process and the high-gradient status of the structure. The status is given by the achieved accelerating gradient and the BDR, which is the number of breakdowns per pulse normalized to the length of the structure $[1 /($ pulse $\mathrm{m})]$. Input power was kept constant in the BDR runs, during the period of operation time when BDR was being evaluated. By counting the breakdown events that occurred in the given period of rf-on time, the BDR was calculated by dividing the number of breakdowns

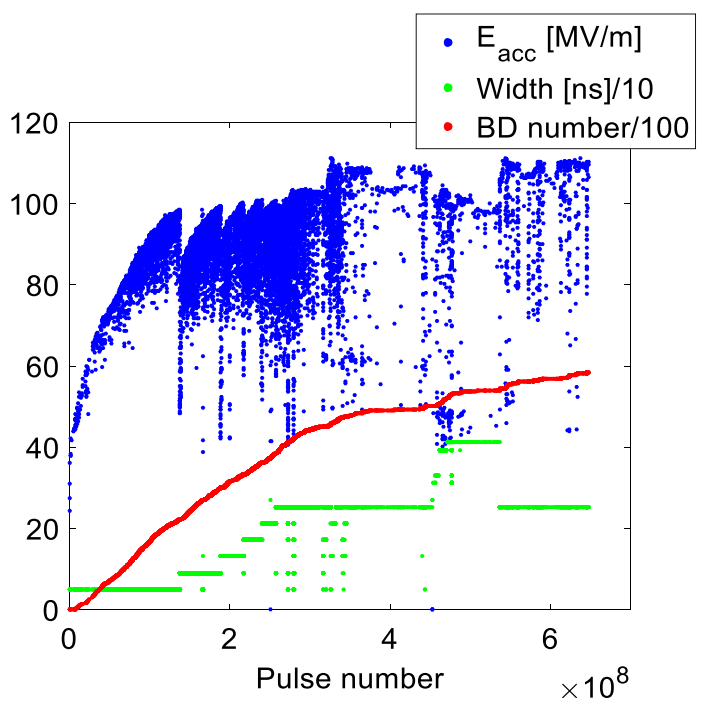

FIG. 6. Summary of the conditioning history. The blue dots denote $E_{\text {acc }}[\mathrm{MV} / \mathrm{m}]$, the green dots denote pulse width [ns] divided by 10 , and the red dots denote breakdown number divided by 100 . 


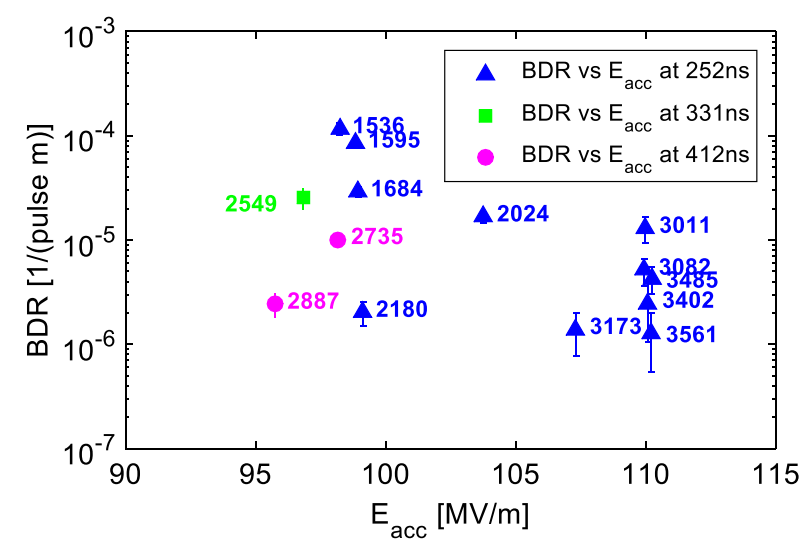

FIG. 7. BDR versus $E_{\text {acc }}$ measured at different rf-on times and pulse widths. The numbers beside the symbols are the integrated operation period in hours since the beginning of the conditioning process until the time of the BDR measurement.

by the number of pulses. Figure 7 shows the BDR measurement result. The 15 BDR measurement runs were conducted in several pulse lengths and field levels. The error bar of the BDR is only statistical and is defined by Formula (1):

$$
E R R_{\mathrm{BDR}}=\frac{1}{\sqrt{\mathrm{Num}_{B D}}} \times \mathrm{BDR},
$$

where $E R R_{\mathrm{BDR}}$ is the error of the $\mathrm{BDR}$ and $\mathrm{Num}_{B D}$ is the number of breakdowns in the BDR measurement. The relative error bars of the low BDR points are high because only a small number of breakdown events occurred even in the long runs.

BDR is strongly dependent on $E_{\text {acc }}$ and rf pulse width. The dependencies that have been observed in many CLIC prototype structures are reported in Ref. [16] and can be approximated with the following relation:

$$
\frac{B D R}{E_{\mathrm{acc}}{ }^{30} \cdot \tau^{5}}=\text { constant }
$$

where $E_{\text {acc }}$ is the unloaded accelerating gradient and $\tau$ is the rf pulse width. Through this scaling law, the BDR can be normalized to $100 \mathrm{MV} / \mathrm{m}$ and $250 \mathrm{~ns}$ to obtain the normalized $\mathrm{BDR}\left(B D R^{*}\right)$ using the following formula:

$$
B D R^{*}=B D R \times\left(\frac{100}{E_{\mathrm{acc}}[\mathrm{MV} / \mathrm{m}]}\right)^{30} \times\left(\frac{250}{\tau[\mathrm{ns}]}\right)^{5},
$$

which is easily deduced from Eq. (2) [42]. The results of $B D R^{*}$, which decreases as the number of rf pulses increases, are shown in Fig. 8. Figure 8 is obtained by replotting the BDR measurement result shown in Fig. 7 as a function of pulse number. The decreasing nature of $B D R^{*}$ (red line in Fig. 8) is fitted by an exponential function of the number of rf pulses:

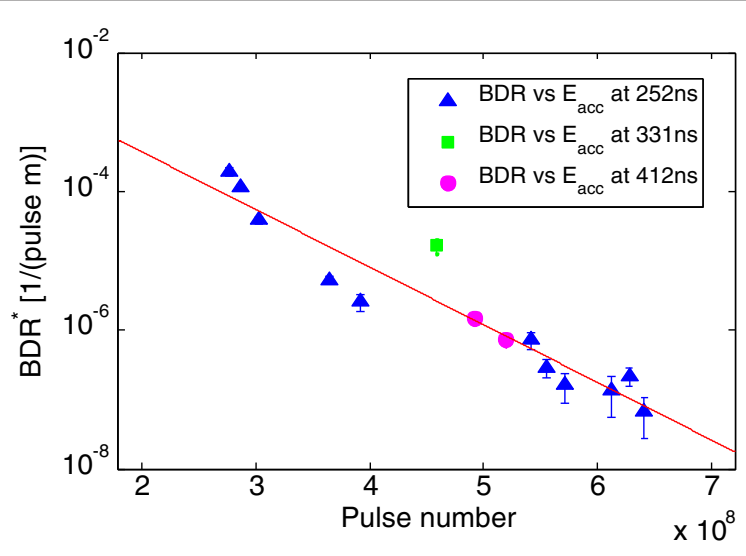

FIG. 8. Normalized BDR versus number of rf pulses.

$$
B D R^{*}=\text { constant } \times \exp \left(-\frac{n}{N_{e}}\right),
$$

where $n$ is the number of rf pulses and $N_{e}$ is defined as the rf pulse evolution factor. This test obtained an rf pulse evolution factor of $5.22 \times 10^{7}$, which is similar to those of the previously tested structures [20].

As expressed in Eq. (2), BDR is strongly dependent on $E_{\text {acc }}$, which is the 30th power of $E_{\text {acc }}$. BDR is measured at different $E_{\text {acc }}$ values to determine its dependence. However, this process requires lengthy runs when the BDR is fairly low, such that the measurement at a given $E_{\text {acc }}$ would cost more than 100 rf-on hours. During the time between the BDR measurements at different $E_{\text {acc }}$ values, additional conditioning of the structure occurs and affects the BDR dependence on $E_{\text {acc }}$. The analysis showed that the rf pulse evolution factor presented previously was applied to compensate for the effect on $E_{\text {acc }}$ dependence, as shown in Fig. 9. The solid symbols denote the raw BDRs obtained from the experimental data, whereas the hollow symbols denote calculated BDRs obtained by applying the rf pulse evolution factor. In the case of $412 \mathrm{~ns}$, the BDR

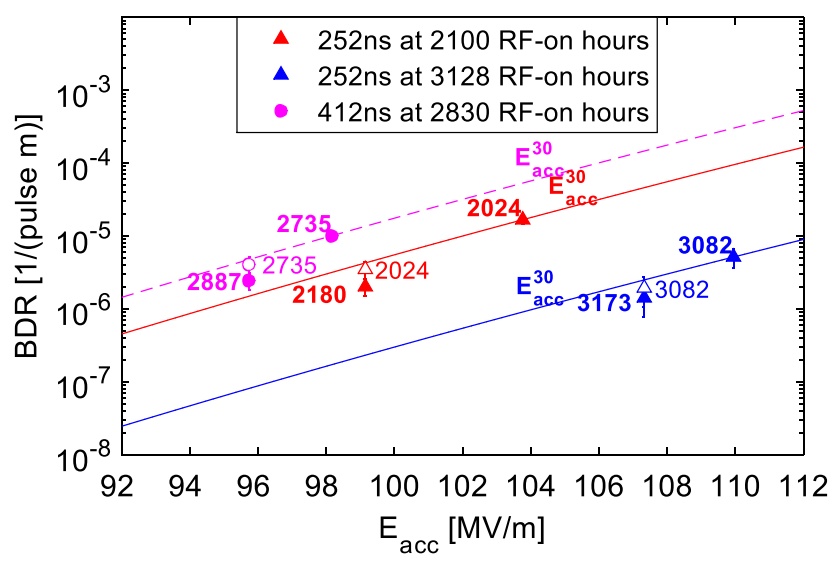

FIG. 9. BDR as a function of $E_{\text {acc }}$ for pulse widths of $252 \mathrm{~ns}$ (red at 2100 rf-on hours and blue at 3128 rf-on hours) and $412 \mathrm{~ns}$ (pink). The reference lines are $E_{\text {acc }}{ }^{30}$. 
measurement at $95.7 \mathrm{MV} / \mathrm{m}$ at $2887 \mathrm{~h}$ was $152 \mathrm{~h}$ $\left(2.74 \times 10^{7} \mathrm{rf}\right.$ pulses) after that at $98.2 \mathrm{MV} / \mathrm{m}$ measured at $2735 \mathrm{~h}$. Therefore, the BDR of the subsequent measurement can be scaled to that of the previous measurement by multiplying the factor of $\exp \left(2.74 \times 10^{7} / N_{e}\right)$ obtained from Eq. (4). Thus, the raw BDRs denoted as solid circles in Fig. 9 were corrected to open circles to estimate the BDR dependence on $E_{\text {acc }}$ at the time of 2735 rf-on hours, as shown in Fig. 9. In the figure, the BDR dependence on $E_{\text {acc }}$ showed a similar power index if this evolution factor is applied to eliminate the time-dependent effect. This practical procedure is employed to analyze the BDR dependency on $E_{\text {acc }}$ at the low BDR case.

The high-gradient status after conditioning of the present structure was $110.2 \mathrm{MV} / \mathrm{m}$ at a BDR of $1.26 \times 10^{-6}$ $1 /$ (pulse $\mathrm{m})$ and a pulse width of $252 \mathrm{~ns}$. This status is equivalent to an estimated BDR of $1.27 \times 10^{-8}$ $1 /$ (pulse $\mathrm{m}$ ) at $100 \mathrm{MV} / \mathrm{m}$ with the pulse width required by the CLIC standard by applying the scaling law $[2,20]$. This achievement ensures that the fabrication technology of Tsinghua University is good enough to proceed with further studies on $X$-band test structures for CLIC. The achieved gradient is approximately $10 \mathrm{MV} / \mathrm{m}$ lower than the gradient of $120 \mathrm{MV} / \mathrm{m}$ at the BDR of the order of $10^{-6}$ $1 /$ (pulse $\mathrm{m}$ ) and the pulse width of $252 \mathrm{~ns}$ obtained in a previous test of a T24 structure at KEK [26]. Improvements to better structure fabrication will be investigated.

\section{Breakdown timing analysis}

The breakdown timing is the time delay between the beginning of the rf pulse and when the breakdown occurs, which is $t_{2}$ shown in Fig. 5. When the structure is operated at a pulse width of $252 \mathrm{~ns}$, the delay can thus range from 0 to $252 \mathrm{~ns}$. The beginning of the collapse of transmitted power was used to obtain the breakdown timing for the subsequent analysis. If no memory effect is observed (in other words, no accumulated surface modifications that eventually lead to a breakdown from one pulse to the subsequent pulse), then the observed $\tau^{5}$ BDR versus pulse width dependence would require that breakdowns be more frequent toward the end of the pulse. The breakdown timing distribution was investigated carefully to evaluate whether the trigger mechanism has such a memory effect.

Histograms of the breakdown timing for two types of $\mathrm{rf}$ breakdowns, i.e., NL-BD and FP-BD, are shown in Fig. 10. The pulse length in these tests was $252 \mathrm{~ns}$. These tests include all of the periods operating at the pulse width of $252 \mathrm{~ns}$, as shown in Fig. 6, including the period from the pulse number $2.57 \times 10^{8}$ to $4.52 \times 10^{8}$ and from the pulse number $5.36 \times 10^{8}$ to $6.47 \times 10^{8}$. Figure 10 shows that the NL-BD events distribute uniformly over the rf pulse. This finding indicates that a memory effect exists and the pulse length of previous pulses affects only the BDR dependence on $\tau$, but not the timing distribution within the pulse width.

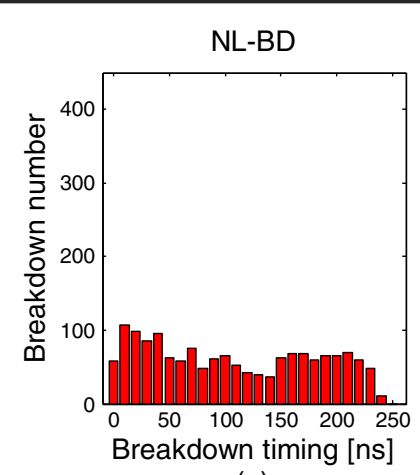

(a)

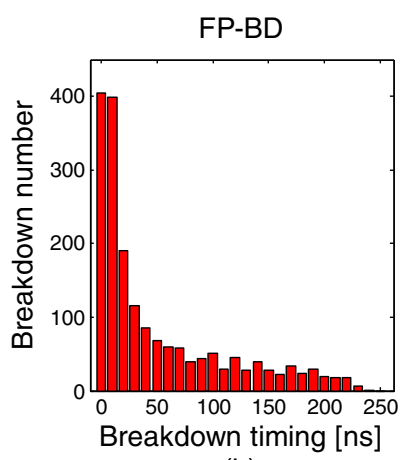

(b)
FIG. 10. Breakdown timing distribution in 252 ns pulse operated at $252 \mathrm{~ns}$ operation. (a) Statistics of 1561 NL-BD events. (b) Statistics of 1859 FP-BD events.

The figure also shows that, in the FP-BDs, the events peak at the beginning of the rf pulse, quickly decay until $50 \mathrm{~ns}$ later, and gradually decrease toward the end of the pulse. Given that the FP-BDs occur at the beginning of the pulse in a lower field level, FP-BDs can be speculated to be caused by surface features, e.g., copper splashes, created by the previous breakdowns.

Figure 11 shows the breakup of the same distribution shown in Fig. 10 into three successive running periods to evaluate the evolution of the timing distribution. The distributions were observed to be the same, i.e., showing no

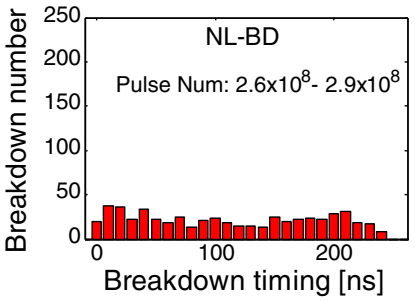

(a)

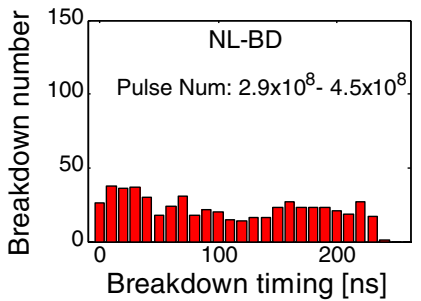

(c)

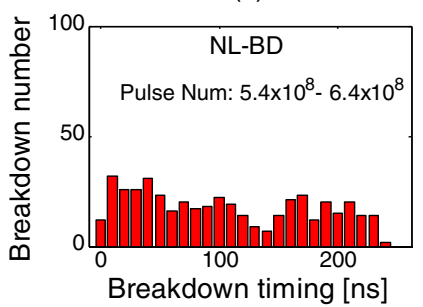

(e)

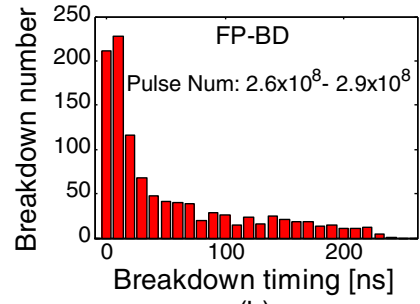

(b)

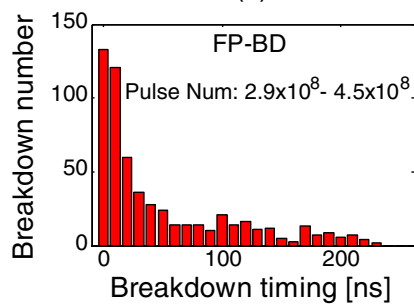

(d)

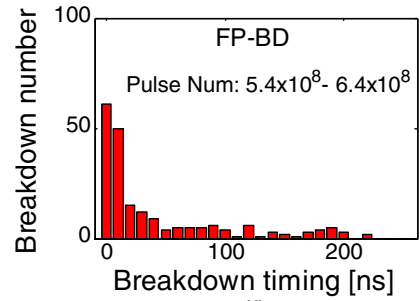

(f)
FIG. 11. Breakdown timing distribution in different processing stages of the 252 ns operation. 


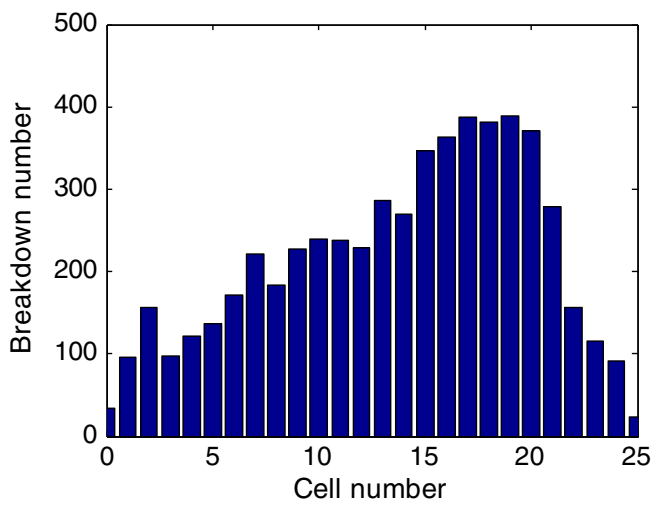

FIG. 12. Distribution of the NL-BD cell location.

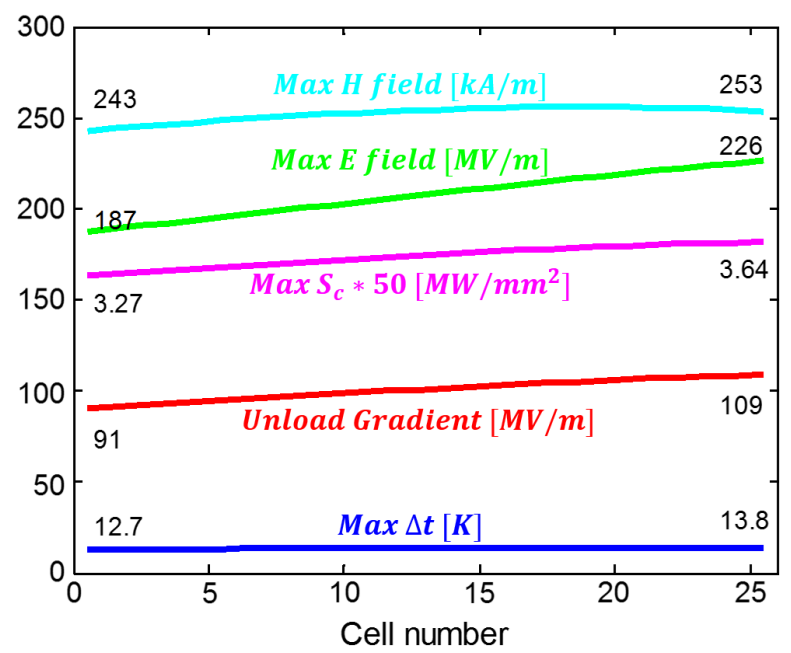

FIG. 13. Distribution of the rf parameters along the structure cells at average unloaded gradient of $100 \mathrm{MV} / \mathrm{m}$ (red: accelerating gradient; blue: pulse temperature rise; green: maximum surface electric field; cyan: maximum surface magnetic field; magenta: maximum modified Poynting vector [15]). evolution. The same phenomena were observed not only in the $252 \mathrm{~ns}$ operation but also in other pulse widths.

\section{Breakdown cell location}

Once a breakdown occurs, incident power is reflected back to the power source and transmission is suppressed, as shown in Fig. 5. This method can be used to calculate the breakdown position and corresponding breakdown cell, with a resolution of approximately one or two structure cells [43]. This technique has been reported in previous studies and references therein [44,45]. The breakdown position distribution in the structure provides important information, which helps in determining the status of the structure during the high-power test. A "hot spot," which dominates the BDR, can occur in a specified cell, e.g., a feature emerges, which increases the BDR locally. No hot spots were observed in the present structure, as shown in Fig. 12, although a broad increase toward the end of the structure occurred. The distributions of the field profiles, including unloaded gradient, maximum surface electric field, maximum magnetic field, maximum modified Poynting vector, and maximum pulse temperature rise are shown in Fig. 13. No obvious relationship between the distributions of the field profiles and the rf breakdown locations was observed, such that the origin of the broad and high BDR region is not understood.

From the two types of breakdowns, i.e., NL-BD and FPBDs, having quite different distributions in breakdown timing, the breakdown cell location was analyzed further. The breakdown cell location of the FP-BDs in run 36, which was an operation period at 252 ns, is shown in Fig. 14. As shown in Fig. 14(a), the FP-BDs occurred uniformly along the structure. By contrast, as shown in Fig. 14(c), the breakdown timing had a sharp peak at the beginning of the pulse. The cell shift is introduced and defined as the breakdown cell difference between the current FP-BD and the breakdown in the previous pulse. The measured distribution of cell shift shown in Fig. 14(b)

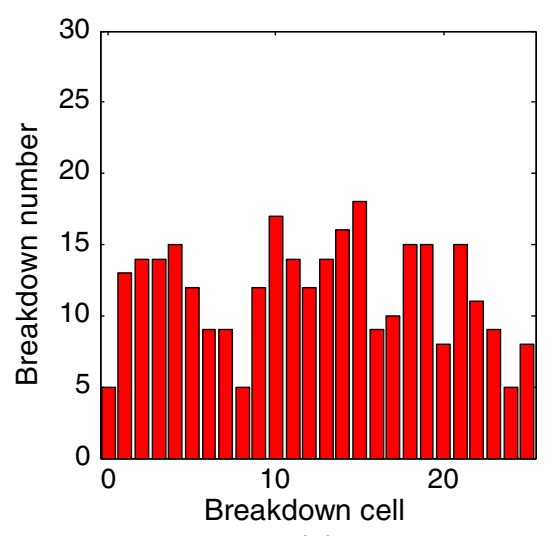

(a)

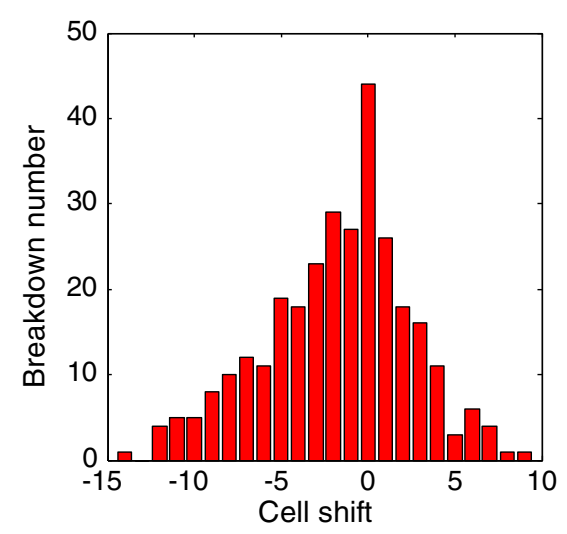

(b)

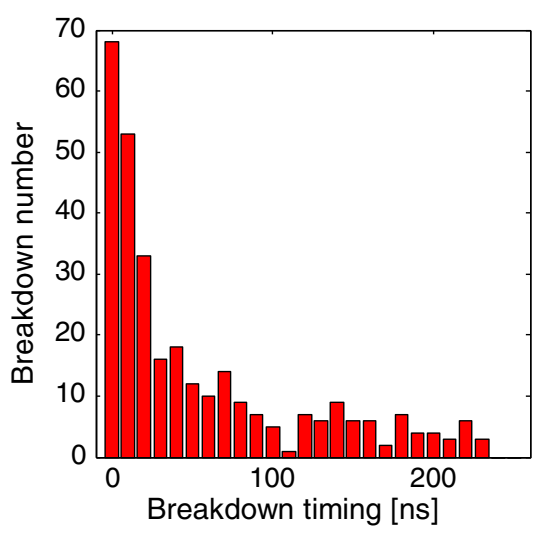

(c)

FIG. 14. Behavior of FP-BDs in run 36. (a) Breakdown position distribution. (b) Cell shift. (c) Breakdown timing. 
indicates that FP-BDs occur near the previous breakdowns. This phenomenon was also observed in other runs.

\section{E. Comparison with other CLIC prototype structures}

In this section, the high-power test results of T24_THU_ \#1 were compared from various points of view with other CLIC prototype structures tested in Nextef. The testing times are shown in Table II to evaluate the statistical significances of data points from different tests. In Table II, "D" refers to damped structure and "R05" refers to corner radius of $0.5 \mathrm{~mm}$ [2].

The structures shown in Table II were all made in collaboration among CERN, KEK, and SLAC. The rf design was made by CERN, and the mechanical design and fabrication of parts were performed by KEK. Details about the design and fabrication of these structures can be found in Refs. [2,13]. The chemical polishing of the parts, the assembly with diffusion bonding and brazing followed by tuning, and the final vacuum baking were performed by SLAC [20]. The high-power tests were conducted at Nextef [21]. The manufacturing flow of T24_THU_\#1 was different, as stated in Sec. II.

\section{Normalized gradient}

From the dependencies of the BDR on $E_{\text {acc }}$ and pulse width, the normalized $E_{\text {acc }}\left(E_{\text {acc }}{ }^{*}\right)$ can be defined as [42]

$$
E_{\mathrm{acc}} * \frac{E_{\mathrm{acc}} \cdot \tau^{1 / 6}}{B D R^{1 / 30}} .
$$

This parameter can be used to compare the experimental data of the two T24 structures, i.e., T24_THU_\#1 and T24_\#3, because the electrical designs of these two structures are exactly the same. The breakdown events occurring during the power ramping stage following the previous breakdown are discarded to derive the envelope of normalized $E_{\text {acc }}$ to obtain the increasing trend of the normalized $E_{\text {acc }}$. The omitted points are kept for other analyses, such as the counting of such breakdowns for temporal BDR estimation.

First, the structures were compared by plotting the normalized $E_{\text {acc }}$ against the rf pulse number, as shown in Fig. 15. The normalized $E_{\text {acc }}$ of T24_THU_\#1 smoothly and continuously increased compared with the $E_{\text {acc }}$ curve shown in Fig. 6. The normalization equation [Eq. (5)] links all of the different pulse width operations together, and

TABLE II. Information of the structures tested in Nextef.

\begin{tabular}{lc}
\hline \hline Structure name & Total rf pulses \\
\hline T24_\#3 & $4.64 \times 10^{8}$ \\
TD24_\#4 & $8.58 \times 10^{8}$ \\
TD24R05_\#2 & $5.16 \times 10^{8}$ \\
TD24R05_\#4 & $6.23 \times 10^{8}$ \\
\hline \hline
\end{tabular}

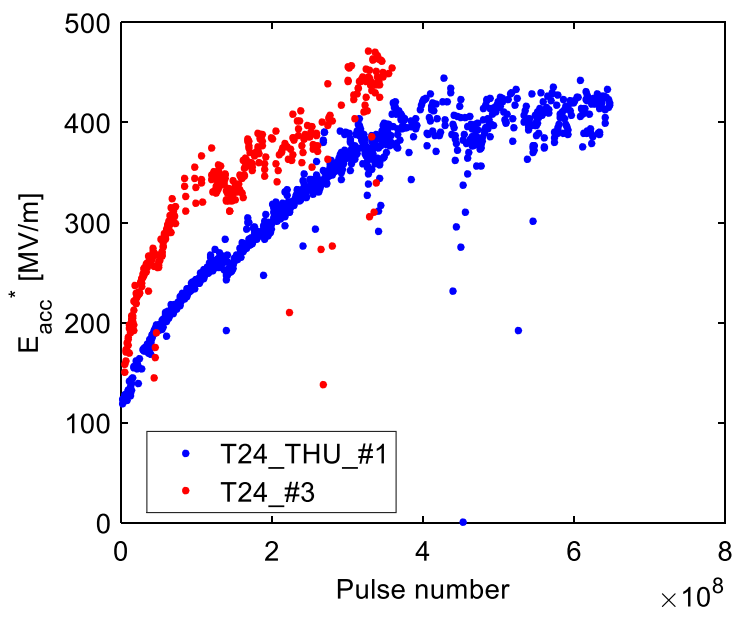

FIG. 15. Comparison of the normalized gradient versus the number of accumulated pulses for T24_THU_\#1 and T24_\#3.

the resulting smooth curve indicates that the structure was conditioned in a smooth manner. In the first comparison plot, T24_\#3 has a faster ramping speed at the beginning and a higher normalized $E_{\text {acc }}$ in the final stage. However, T24_THU_\#1 required more rf-on time to reach the same level as T24_\#3.

Second, these two structures were compared by plotting the normalized $E_{\text {acc }}$ against the breakdown number shown in Fig. 16. In contrast to the first comparison, the two curves shown in Fig. 16 vary significantly. T24_THU_\#1 accumulates many more breakdowns to reach the same normalized $E_{\text {acc }}$ as T24_\#3, indicating that T24_THU_\#1 has a higher BDR during the conditioning process.

Reference [42] points out that the conditioning state improves with the number of rf pulses, but not with the number of breakdowns, and the data from this structure broadly support this observation. A large divergence is

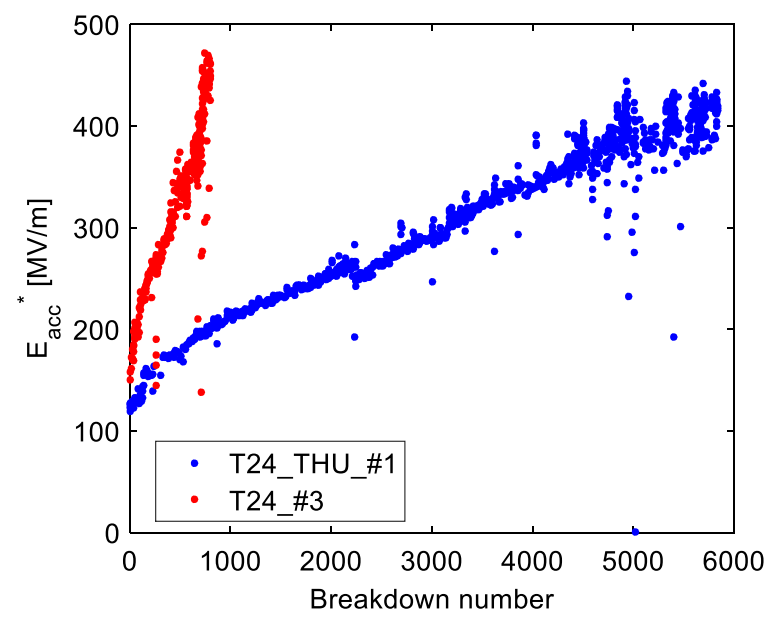

FIG. 16. Comparison of the normalized gradient versus the number of accumulated breakdowns for T24_THU_\#1 and T24_\#3. 


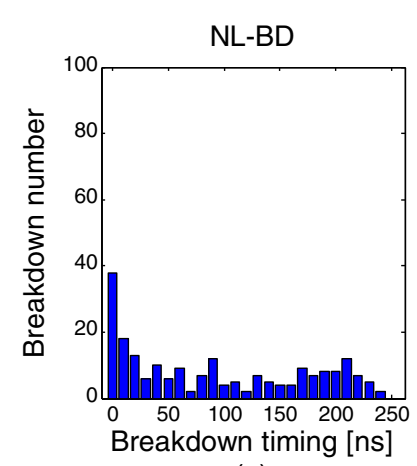

(a)

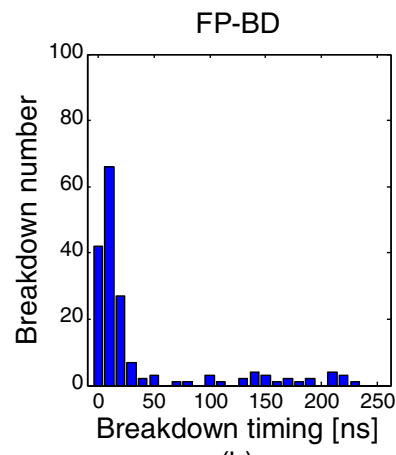

(b)

FIG. 17. Breakdown timing distribution of T24_\#3.

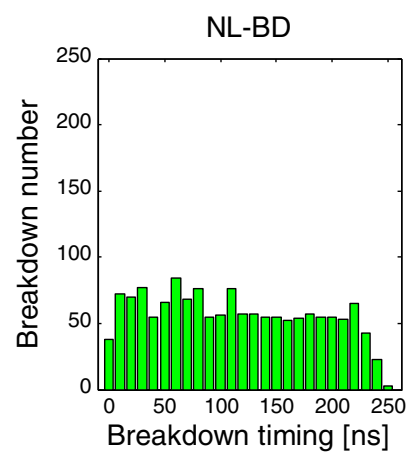

(a)

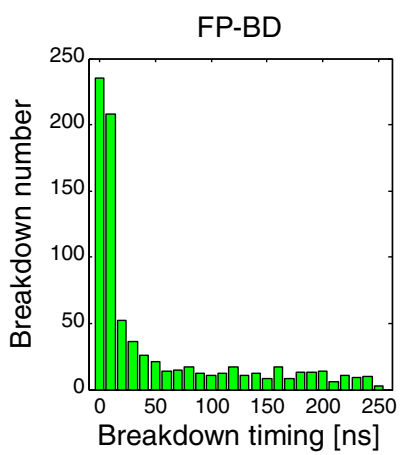

(b)

FIG. 18. Breakdown timing distribution of TD24_\#4.

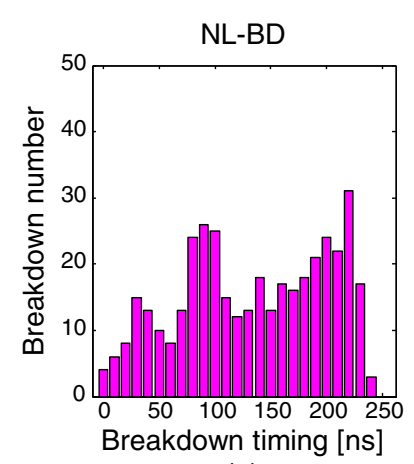

(a)

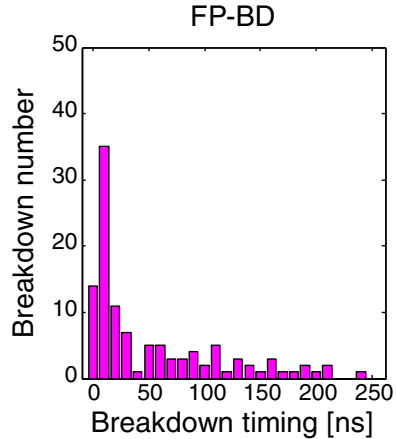

(b)

FIG. 19. Breakdown timing distribution of TD24R05_\#2.

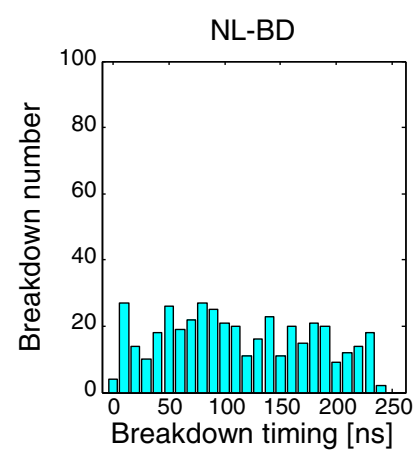

(a)

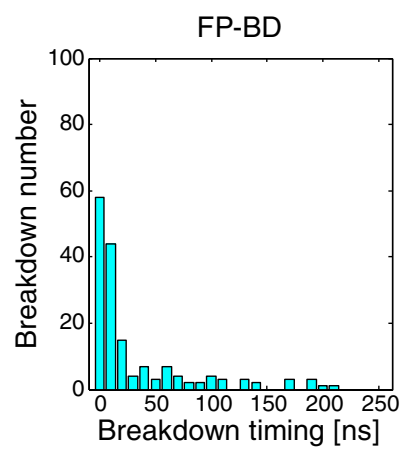

(b)

FIG. 20. Breakdown timing distribution of TD24R05_\#4.

TABLE III. Number of breakdowns of the tested structures.

\begin{tabular}{lcc}
\hline \hline Structure name & Number of NL-BDs & Number of FP-BDs \\
\hline T24_\#3 & 210 & 176 \\
TD24_\#4 & 1477 & 811 \\
TD24R05_\#2 & 392 & 113 \\
TD24R05_\#4 & 425 & 166 \\
\hline \hline
\end{tabular}

observed in the plot of the normalized $E_{\text {acc }}$ versus the accumulated breakdowns. T24_\#3 has a faster conditioning speed in the plot of the normalized $E_{\text {acc }}$ versus the rf pulses. However, the two curves shown in Fig. 15 are approximately similar to each other. This finding is consistent with the conclusion reached in Ref. [42]. The difference of the conditioning speed is speculated to be caused by the differences in machining, assembly, and baking. However, an unknown factor limits the highest normalized $E_{\text {acc }}$ of T24_THU_\#1 in the final stage and should be further investigated.

\section{Breakdown timing}

The four structures described previously were analyzed to determine whether the breakdown timing feature observed in T24_THU_\#1 was reproduced in other tests. Figures 17, 18, 19, 20 show the breakdown timing distributions of these structures. The number of breakdowns at $252 \mathrm{~ns}$ pulse width operation of the four structures are presented in Table III. The same feature that NL-BDs have a uniform distribution in the pulse, whereas the FPBDs have a high breakdown probability at the beginning of the rf pulse was observed in all cases.

\section{DISCUSSION}

The origin of the two types of distributions is considered. Conditioning is a process that modifies the cavity surface using the rf fields [22]. One explanation for the occurrence of a breakdown at a particular place is the presence of a protrusion, which results in field enhancement leading to enhanced electron emission. The emission currents lead to the breakdown. Such emitters "tips" are melted by the breakdown plasma. The resulting features, often called craters, look like splashes and have new protrusions, which may have a higher field enhancement factor than the original feature and become new emitters. Thus, the subsequent rf pulse power should be reduced by the control algorithm. Experimental and theoretical studies on both pulsed dc and rf indicate that there might be a limit value which is constant and depends only on the electrode material for the local field $[23,46,47]$. Once the local field goes beyond this limit, the breakdown will be triggered. The local field at the new emitter may exceed the breakdown limit at the beginning of the subsequent rf pulse and trigger the FP-BD. 


\section{CONCLUSION}

An $X$-band 24-cell CLIC prototype traveling-wave accelerator structure called T24_THU_\#1 has been successfully fabricated at Tsinghua University. A high-power test was conducted at the Nextef facility in KEK, and the test demonstrated that the structure can operate at 110.2 MV/m at a BDR of $1.26 \times 10^{-6} 1 /$ (pulse $\mathrm{m}$ ) and a pulse width of $252 \mathrm{~ns}$. The fabrication capability of Tsinghua University for $X$-band high-gradient structures has been validated by comparing the results with previously tested CLIC prototype structures. The high-power performance of T24_THU_\#1 was within 10\% in the gradient of a previously tested structure of an identical rf design, indicating that the fabrication quality was approximately the same, but a certain margin of improvement still remains. A second T24 structure will be built by Tsinghua University to determine whether the fabrication quality can be improved, and the same operation and analysis will be applied to compare structures and further validate the results presented here.

An rf pulse evolution constant of the order of $10^{7}$ was observed from the conditioning rate of the structure and other CLIC prototype structures tested in Nextef. The rf pulse evolution constant can be applied to compensate for the conditioning effect in the analysis of the BDR dependence on $E_{\text {acc }}$, resulting in the simple behavior of the BDR as a function of the accelerating gradient. Different features of two types of breakdowns, i.e., NL-BDs and FP-BDs, were observed during the experiment. The breakdown timing distribution of NL-BDs is uniform, whereas that of FP-BDs peaked at the beginning of the rf pulse. The feature left behind previous breakdown, which has a higher field enhancement, is speculated to be one of the trigger sources of the FP-BD occurring at the beginning of the rf pulse because it exceeded the local field limitation.

\section{ACKNOWLEDGMENTS}

This work was supported by the National Natural Science Foundation of China (Grant No. 11135004). The experimental program had also been supported as one of the collaborations of the CLIC under the agreement between Tsinghua University and CLIC and that between KEK and CERN (ICA-JP-0103). The authors thank the KEK electron-positron injector group for supporting the long-term operation over a year.

[1] A. Degiovanni, S. Doebert, W. Farabolini et al., Highgradient test results from a CLIC prototype accelerating structure: TD26CC, in Proceedings of the 5th International Particle Accelerator Conference, IPAC-2014, Dresden, Germany, 2014 (JACow, Dresden, Germany, 2014), pp. 2285-2287.
[2] M. Aicheler, P. Burrows, M. Draper, T. Garvey, P. Lebrun, K. Peach, N. Phinney, H. Schmickler, D. Schulte, and N. Toge, CLIC Conceptual Design Report CERN 007, 2012.

[3] A. Grudiev and W. Wuensch, Design of the CLIC main linac accelerating structure for CLIC conceptual design report, in Proceedings of the 25th International Linear Accelerator Conference, LINAC-2010, Tsukuba, Japan, 2010 (JACow, Tsukuba, Japan, 2010), pp. 211-213.

[4] T. Shintake, The choke mode cavity, Jpn. J. Appl. Phys. 31, L1567 (1992).

[5] J. Shi et al., Design of a choke-mode damped accelerating structure for CLIC main linac, in Proceedings of the 2nd International Particle Accelerator Conference, IPAC2011, San Sebastián, Spain, 2011 (JACow, San Sebastián, Spain, 2011), pp. 113-115.

[6] H. Zha, J. Shi, H. Chen, A. Grudiev, W. Wuensch, C. Tang, and W. Huang, Choke-mode damped structure design for the Compact Linear Collider main linac, Phys. Rev. ST Accel. Beams 15, 122003 (2012).

[7] X.W. Wu, J. Shi, H. B. Chen et al., High gradient properties of a CLIC prototype accelerating structure made by Tsinghua University, in Proceedings of the 7th International Particle Accelerator Conference, IPAC2016, Busan, Korea, 2016 (JACow, Busan, Korea 2016), pp. 3874-3877.

[8] V. F. Khan, Ph.D. thesis, University of Manchester, 2010.

[9] V.F. Khan, A. D'Elia, R. M. Jones, A. Grudiev, W. Wuensch, G. Riddone, and V. Soldatov, Wakefield and surface electromagnetic field optimisation of manifold damped accelerating structures for CLIC, Nucl. Instrum. Methods Phys. Res., Sect. A 657, 131 (2011).

[10] R. M. Jones, Wakefield suppression in high gradient linacs for lepton linear colliders, Phys. Rev. ST Accel. Beams 12, 104801 (2009).

[11] H. Zha and A. Grudiev, Design and optimization of Compact Linear Collider main linac accelerating structure, Phys. Rev. Accel. Beams 19, 111003 (2016).

[12] X. W. Wu et al., High power test of X-band single cell HOM-free choke-mode damped accelerating structure made by Tsinghua University, in Proceedings of the 7th International Particle Accelerator Conference, IPAC2016, Busan, Korea (Ref. [7]), pp. 3881-3883.

[13] T. Higo et al., Advances in X-band TW accelerator structures operating in the $100 \mathrm{MV} / \mathrm{m}$ regime, in Proceedings of the 1st International Particle Accelerator Conference, IPAC-2010, Kyoto, Japan, 2010 (JACow, Kyoto, Japan, 2010), pp. 3702-3704.

[14] R. Zennaro et al., Design and fabrication of CLIC test structures, in Proceedings of Linear Accelerator Conference, LINAC-2008, Victoria, BC, Canada, 2008 (JACow, Victoria, BC, Canada, 2008), pp. 533-535.

[15] A. Grudiev, in Proceedings of the 4th Annual X-band Structure Collaboration Meeting, CERN, 2010, http:// indico.cern.ch/conferenceDisplay.py?confId $=75374$.

[16] A. Grudiev, S. Calatroni, and W. Wuensch, New local field quantity describing the high gradient limit of accelerating structures, Phys. Rev. ST Accel. Beams 12, 102001 (2009).

[17] S. Matsumoto, M. Akemoto, S. Fukuda, T. Higo, N. Kudoh, H. Matsushita, H. Nakajima, T. Shidara, K. Yokoyama, and M. Yoshida, NEXTEF: 100MW X-band 
test facility in KEK, in Proceedings of the 11th European Particle Accelerator Conference, EPAC-2008, Genoa, Italy, 2008 (JACow, Genoa, Italy, 2008), pp. 2740-2742.

[18] S. Fukuda et al., R\&D plan for RF power source of KEK GLCTA, in Proceedings of the First Annual Meeting of the Accelerator Society of Japan (Particle Accelerator Society of Japan, Funabashi, Japan, 2004), pp. 75 (in Japanese).

[19] J. P. Delahaye, Towards CLIC feasibility, in Proceedings of the 1st International Particle Accelerator Conference, IPAC-2010, Kyoto, Japan, 2010 (Ref. [13]), pp. 4769-4773.

[20] S. Matsumoto, T. Abe, Y. Higashi, T. Higo, and Y. Du, High gradient test at Nextef and high-power long-term operation of devices, Nucl. Instrum. Methods Phys. Res., Sect. A 657, 160 (2011).

[21] T. Higo et al., Comparison of high gradient performance in varying cavity geometries, in Proceedings of the 4th International Particle Accelerator Conference, IPAC2013, Shanghai, China, 2013 (JACoW, Shanghai, China, 2013), pp. 2741-2743.

[22] J. W. Wang and G. A. Loew, SLAC Report No. 7684, 1997.

[23] A. Descoeudres, Y. Levinsen, S. Calatroni, M. Taborelli, and W. Wuensch, Investigation of the dc vacuum breakdown mechanism, Phys. Rev. ST Accel. Beams 12, 092001 (2009).

[24] V. A. Dolgashev and S. G. Tantawi, SLAC Report No. 10175, 2003.

[25] K. Nordlund and F. Djurabekova, Defect model for the dependence of breakdown rate on external electric fields, Phys. Rev. ST Accel. Beams 15, 071002 (2012).

[26] T. Higo et al., Recent high-gradient test result at KEK, in Proceedings of the 2012 Linear Collider Workshop, LCWS2012, Arlington, VA, 2012 (LCWS2012, Arlington, VA, 2012).

[27] V. Dolgashev et al., in Proceedings of the 2013 High Gradient Workshop (ICTP, Italy, 2013).

[28] B. J.Munroe, A. M. Cook, M. A. Shapiro, R. J. Temkin, V. A. Dolgashev, L. L. Laurent, J. R. Lewandowski, A. D. Yeremian, S. G. Tantawi, and R. A. Marsh, High power breakdown testing of a photonic band-gap accelerator structure with elliptical rods, Phys. Rev. ST Accel. Beams 16, 012005 (2013).

[29] F. Wang, C. Adolphsen, and C. Nantista, Performance limiting effects in X-band accelerators, Phys. Rev. ST Accel. Beams 14, 010401 (2011).

[30] F. Wang, C. Adolphsen, and C. Nantista, Initial high power test results of an X-band dual-moded coaxial cavity, in Proceedings of the 24th Particle Accelerator Conference, PAC-2011, New York, USA, 2011 (IEEE, New York, USA, 2011), pp. 1094-1096.

[31] A. D. Yeremian, V. A. Dolgashev, J. Nielson, and S. G. Tantawi, Dual mode accelerating cavity to test rf break- downs dependence on rf magnetic fields, in Proceedings of the 2nd International Particle Accelerator Conference, IPAC-2011, San Sebastián, Spain, 2011 (Ref. [5]), pp. 247-249.

[32] H. Timko, M. Aicheler, P. Alknes, S. Calatroni, A. Oltedal, A. Toerklep, M. Taborelli, W. Wuensch, F. Djurabekova, and K. Nordlund, Energy dependence of processing and breakdown properties of $\mathrm{Cu}$ and Mo, Phys. Rev. ST Accel. Beams 14, 101003 (2011).

[33] L. Laurent, S. Tantawi, V. Dolgashev, C. Nantista, Y. Higashi, M. Aicheler, S. Heikkinen, and W. Wuensch, Experimental study of rf pulsed heating, Phys. Rev. ST Accel. Beams 14, 041001 (2011).

[34] VDL company, http://www.vdletg.com/.

[35] GLC Project Report, KEK-Report-2003-7.

[36] J. Wang and T. Higo, Accelerator structure development for NLC/GLC, in International Committee for Future Accelerators (ICFA): Beam Dynamics Newsletter No. 32 (2003), pp. 27-46.

[37] J. Shi et al., Recent X-band activities at Tsinghua University, in Proceedings of the 2015 CLIC Workshop, Geneva, Switzerland, 2015, (CLIC Workshop 2015, Geneva, Switzerland, 2015).

[38] J. Shao et al., Observation of Field-Emission Dependence on Stored Energy, Phys. Rev. Lett. 115, 264802 (2015).

[39] J. Shao et al., In Situ Observation of Dark Current Emission in a High Gradient rf Photocathode Gun, Phys. Rev. Lett. 117, 084801 (2016).

[40] C. Adolphsen, SLAC Report No. 9906, 2003.

[41] V.A. Dolgashev et al., Study of basic rf breakdown phenomena in high gradient vacuum structures, in Proceedings of the 25th International Linear Accelerator Conference, LINAC-2010, Tsukuba, Japan, 2010 (Ref. [3]), pp. 1043-1047.

[42] A. Degiovanni, W. Wuensch, and J. G. Navarro, Comparison of the conditioning of high gradient accelerating structures, Phys. Rev. Accel. Beams 19, 032001 (2016).

[43] W. Wuensch, A. Degiovanni, S. Calatroni, A. Korsbäck, F. Djurabekova, R. Rajamäki, and J. Giner-Navarro, Statistics of vacuum breakdown in the high-gradient and low-rate regime, Phys. Rev. Accel. Beams 20, 011007 (2017).

[44] B. J. Woolley, Ph.D. thesis, 2015.

[45] R. Rajamaki, Master's thesis, Aalto University, 2016.

[46] H. Timko, K. Matyash, R. Schneider, F. Djurabekova, K. Nordlund, A. Hansen, A. Descoeudres, J. Kovermann, A. Grudiev, W. Wuensch, S. Calatroni, and M. Taborelli, A one-dimensional particle-in-cell model of plasma build-up in vacuum arcs, Contrib. Plasma Phys. 51, 5 (2011).

[47] J. Shao, Ph.D. thesis, Tsinghua University, 2016. 